Pacific Northwest

National Laboratory

Operated by Battelle for the

U.S. Department of Energy

\title{
Safety Report for Raman Spectrometry: Safety Evaluation and Search Algorithm Enhancement
}

S.D. Harvey

B.W. Wright

April 2002

Prepared for the U.S. Department of Energy under Contract DE-AC06-76RL01830

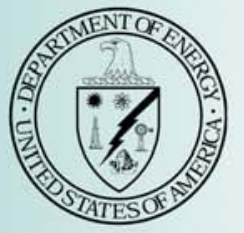




\title{
DISCLAIMER
}

This report was prepared as an account of work sponsored by an agency of the United States Government. Neither the United States Government nor any agency thereof, nor Battelle Memorial Institute, nor any of their employees, makes any warranty, express or implied, or assumes any legal liability or responsibility for the accuracy, completeness, or usefulness of any information, apparatus, product, or process disclosed, or represents that its use would not infringe privately owned rights. Reference herein to any specific commercial product, process, or service by trade name, trademark, manufacturer, or otherwise does not necessarily constitute or imply its endorsement, recommendation, or favoring by the United States Government or any agency thereof, or Battelle Memorial Institute. The views and opinions of authors expressed herein do not necessarily state or reflect those of the United States Government or any agency thereof.

\author{
PACIFIC NORTHWEST NATIONAL LABORATORY \\ operated by \\ BATTELLE \\ for the \\ UNITED STATES DEPARTMENT OF ENERGY \\ under Contract DE-AC06-76RL01830
}

Printed in the United States of America

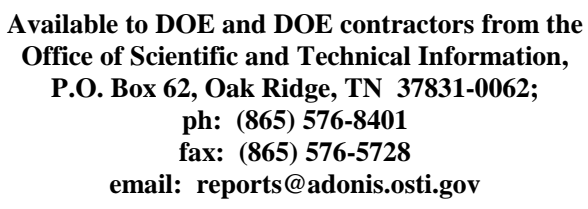

Available to the public from the National Technical Information Service, U.S. Department of Commerce, 5285 Port Royal Rd., Springfield, VA 22161 ph: (800) 553-6847 fax: $(703) 605-6900$

email: orders@ntis.fedworld.gov

online ordering: http://www.ntis.gov/ordering.htm 


\section{Safety Report}

for

\section{Raman Spectrometry: Safety Evaluation and Search Algorithm Enhancement}

Scott D. Harvey

Bob W. Wright

Prepared for

The Hazardous Material Response Unit

Federal Bureau of Investigation

Under a Related Services Agreement

With the U.S. Department of Energy

Under Contract DE-AC06-76RLO 1830

Pacific Northwest National Laboratory

Richland, Washington 99352

April 2002 


\section{CONTENTS}

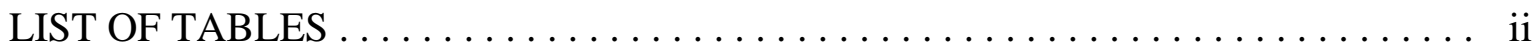

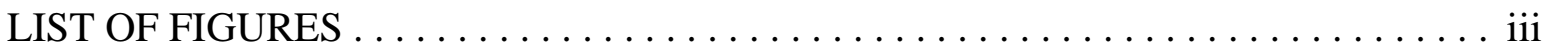

EXECUTIVE SUMMARY $\ldots \ldots \ldots \ldots \ldots \ldots \ldots \ldots \ldots \ldots \ldots \ldots \ldots$

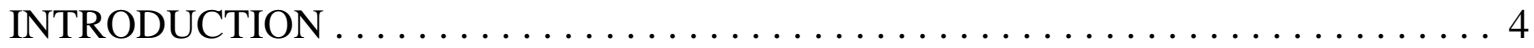

EXPERIMENTAL STUDIES $\ldots \ldots \ldots \ldots \ldots \ldots \ldots \ldots \ldots \ldots \ldots \ldots$

Heat Transfer to Colored Surfaces. ......................... 5

Experimental Design and Set-up ..................... 5

Time Course Experiment . . . . . . . . . . . . . . . . . 10

Thermal Imaging Results $\ldots \ldots \ldots \ldots \ldots \ldots \ldots \ldots \ldots \ldots \ldots \ldots$

Solvent Flammability . . . . . . . . . . . . . . . . . . . . . . . . 13

Preparation for Flammability Experiments . . . . . . . . . . . . 13

Parameters Effecting Solvent Ignition Potential .............. 13

Solvent Flammability on Graphite-Coated Surfaces ............. 15

Uncoated Porcelain and Aluminum, As Well As Anodized Aluminum

Surfaces ......................................... 17

Bulk Solvent Heating ................................ 17

Deflagration of Explosives and Propellants ...................... 20

Propellants .................................... 20

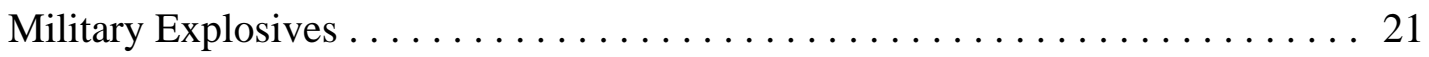

Neat Materiel ...................................... 21

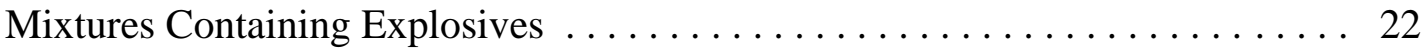

Shock Sensitive Explosives . . . . . . . . . . . . . . . . 26

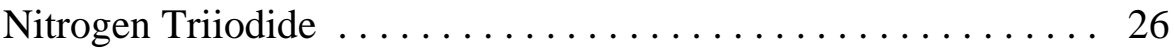

Trinitroglycerin ......................... 26

Deflagration of GunPowders (Smokeless, Black, and Pyrodex Powders). . . . . . 28

CONCLUSIONS AND RECOMMENDATIONS . . . . . . . . . . . . . 30

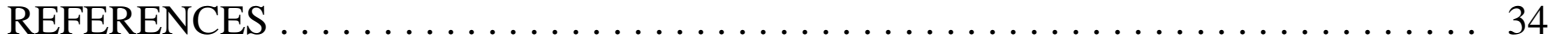




\section{TABLES}

$1 \quad$ Temperatures for Different Colors . . . . . . . . . . . . . . . . . . . . 11

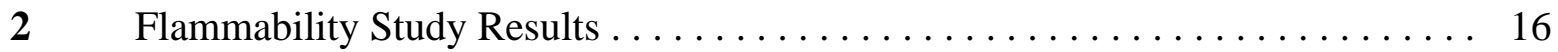

3 Deflagration Results For Neat Explosives . . . . . . . . . . . . . . . . . . 21

4 Deflagration Results for Explosives Mixed with ADS775PP . .............. 23

5 Deflagration Results for RDX Mixed with Matrix Materials ............. 25

6 Deflagration Results for RDX Mixed With Aluminum, Iron Oxide, and Thermite Powders. . . . . . . . . . . . . . . . . . . . . . . . . . . . 25

$7 \quad$ Gunpowder Designations and Particle Sizes $\ldots \ldots \ldots \ldots \ldots \ldots \ldots \ldots$

\section{LIST OF FIGURES}

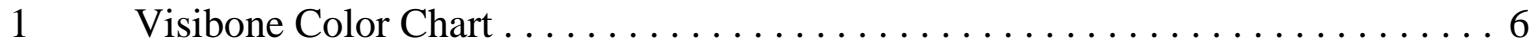

$2 \quad$ VisiBone Chart Strip on Insulated Optical Mount $\ldots \ldots \ldots \ldots \ldots \ldots \ldots$

3 Thermal Imaging Experimental Set-up. . . . . . . . . . . . . . . . . 8

$4 \quad$ Emissivity Determination. ................................ 9

$5 \quad$ Representative Temporal Profiles for Various Colors .................. 10

$6 \quad$ Relative Hazards for Analysis of VisiBione Colors. . . . . . . . . . . . . . . 12

$7 \quad$ Photograph of Aluminum and Porcelain Blocks . . . . . . . . . . . . . . . . . . 14

8 Spectrum of ADS775PP in Methanol ...................... 18

$9 \quad$ Bulk Solution Heating Results . . . . . . . . . . . . . . . . . . . 20

10 Raman Spectrum of Trinitroglycerin ........................ 27

11 Gunpowder Sensitivity Toward the Raman Laser . . . . . . . . . . . . . . . 30 
PNNL-13942

\section{Executive Summary}

This study elucidates the critical safety hazards associated with using a 300-mW 785-nm near-IR Raman laser for sample analysis. Most of the safety concerns are associated with excessive sample heating resulting from sample illumination. Thermography experiments were designed to quantitatively assess which visible surface colors heat most when exposed to the Raman laser. Studies were also conducted with a flammable organic solvent mixed with a nearIR absorbing dye to evaluate the magnitude of solution heating. The next series of experiments examined the tendency of propellants and military explosives, as well as mixtures of these explosives with IR-opaque dyes or matrix materials, to deflagrate upon illumination. Several neat shock-sensitive explosives were also examined to test whether detonation could be induced by laser exposure. Finally, a study was conducted to define the ignition sensitivity of various gunpowders (smokeless, black and Pyrodex powders) toward the Raman laser. These studies are described in more detail in the summary paragraphs below.

Thermography experiments measured the thermal consequence of illuminating a wide range of colors with the Raman laser. Safety concerns are largely defined by the amount of thermal energy imparted to the sample by absorption of the laser light; the higher the absorbed energy, the higher the probability of sample ignition or deflagration. This experiment exposed color patches with defocused laser light while monitoring the increase of the surface temperature with an IR camera. The color swatches chosen were from a web design color chart that contained 216 colors. Each color had unambiguous identifiers that allowed cross correlation with other color charts. Temperatures achieved after 12 seconds of illumination (after thermal equilibrium had been reached) are presented for all 216 colors. Final temperatures ranged from $146.8^{\circ} \mathrm{C}$ for black to $28.0^{\circ} \mathrm{C}$ for white. In general, darker colors showed higher temperatures and, therefore, a higher thermal hazard. A simplified hazard chart was generated that shows color chart positions with increasing thermal risk represented by the progression of yellow through orange and finally red. A laminated color chart included with this report allows a field agent to match a sample color with the chart to quickly identify the thermal hazard associated with the sample by referencing Figures and Tables contained in this report.

Further studies focussed on defining hazards associated with analysis of flammable organic solvents. A variety of solvents, including extremely volatile solvents (i.e., diethyl ether) were studied, on a variety of surfaces, including low thermal conductivity glass and porcelain surfaces coated with IR-opaque paint. Although a few solvents exhibited observable heating effects, as evidenced by enhanced evaporation and even localized boiling in a few cases, solvent ignition was not observed. Related studies examined the tendency of an organic solvent solution that contained a near-IR absorbing dye to heat when illuminated with the Raman laser. The results of this study showed classical heating curves; however, the magnitude of heating was relatively small (i.e., less than $5.6^{\circ} \mathrm{C}$ over $10 \mathrm{~min}$ for the highest dye concentration) and would not be expected to cause problems during Raman analysis. Overall, it was concluded that analysis of flammable solvents by Raman spectroscopy posed a minimal safety risk. 
Next, neat explosives (TNT, tetryl, RDX, HMX, BTTN, and PETN) were examined on a variety of surfaces including aluminum, graphite-coated porcelain, and black construction paper. Illumination of neat military explosives or propellants with the near-IR laser failed to initiate sustainable delfagration on the aluminum or graphite-coated porcelain surfaces. A few explosives (i.e., TNT, tetryl, and RDX) could be coaxed to deflagrate on black construction paper; however, this combustion was difficult to achieve and was not vigorous or self-sustained.

Two shock sensitive explosives, nitrogen triiodide and trinitroglycerin, were also examined during this study. The nitrogen triiodide consistently detonated when illuminated at a laser power well below maximum intensity. Due to the black color of this material, this behavior is not surprising. Trinitroglycerin, a material that is not expected to strongly absorb near-IR radiation, was illuminated for prolonged periods on a variety of surfaces without any observable effects.

Further experiments examined mixtures of explosives (TNT, tetryl, RDX, HMX and PETN) with a near-IR absorbing dye, or mixtures of RDX with various matrix materials. Experiments that examined explosive/dye mixtures showed enhanced reactivity compared to the dye control. With the exception of PETN, the differences between the dye control and explosive mixtures were subtle. Reactions typically consisted of igniting an ember (ember was visible through the laser safety goggles) in the mixture or, at most, initiating deflagration of an individual explosive flake (for TNT) or pellet (for tetryl). On the other hand, the PETN/dye mixture gave a vigorous and almost self-sustained burn accompanied by copious smoke.

Additional experiments were carried out on mixtures of RDX with various matrix materials including iron oxide pellets, a carbon-based chromatographic molecular sieve material (Carboxen), graphite, soil, and several IR absorbing dyes. Again, reactions in the RDXcontaining mixtures were uniformly more vigorous than the matrix material controls. In most cases, the differences between the RDX-containing mixtures and the matrix controls were subtle; the mixtures did not react violently or achieve sustained deflagration. The RDX mixture with iron oxide pellets did, however, give a violent self-sustained deflagration. Coating of RDX powder on the relatively large iron oxide particles seemed to be a critical prerequisite for this self sustained reaction. Less violent reaction occurred for RDX mixed with a fine iron oxide powder (see below).

Because of the violent reaction achieved with the iron oxide pellets mixed with RDX, we examine mixtures of RDX with finely divided iron oxide or aluminum powders, or a mixture of iron oxide and aluminum (a composition known as thermite). RDX was observed to delagrate in the iron oxide powder mixture, however, this reaction was not self sustained and was nowhere near as vigorous as observed for the larger iron oxide pellets. Illumination of thermite, both with and without RDX, yielded bright flashes of white light indicating localized ignition of the thermite material itself, however, this ignition was not self sustained. The bright flashes obscured observation of any RDX deflagration.

Our conclusion regarding the safety of analyzing explosives, explosive mixtures, and propellants was complicated by the fact that these reactions were not interpretable as an all or nothing reaction. Although one may consider it relatively safe to interrogate milligram quantities of an unknown sample, even if the sample heats to the point of emitting wisps of smoke, the 
same experiment surly would pose an unacceptable risk if kilogram or larger sample sizes were involved. A theme that has emerged from our studies is that color is a fundamentally important parameter. All light colored samples can be successfully probed without causing safety concerns. If, on the other hand, there is a possibility the material is an explosive and the color falls within the high risk area of the color chart, it is prudent to limit the amount of sample subjected to analysis to less than approximately $25 \mathrm{mg}$. Solid samples should not be interrogated on black backgrounds regardless of the amount or color of the sample.

The final experimental series examined gunpowders in the smokeless, black, or Pyrodex classes. All gunpowders consistently deflagrated upon exposure to the near-IR laser. For the smokeless class, we examined two flake powders and a cylindrical canister powder, all of which had different burn rates. For the black and Pyrodex classes, we examined two representatives for each that had different particle sizes. The results clearly showed the smokeless powder had a higher sensitivity toward near-IR laser-induced ignition than the black or the Pyrodex powders. There were no obvious differences in ignition sensitivity between any of the representatives within each powder class. It was hypothesized that the higher sensitivity exhibited by smokeless powders was due to the nitroglycerin base. All gunpowders have a characteristic black or gray color. Our thermography experiments indicate that gray and black represent the highest risk colors for analysis with the near-IR laser. Our recommendation is to avoid analysis of any sample that is either gray or black. 
PNNL-13942

\section{Introduction}

Use of high-powered lasers for chemical analysis pose certain well known health hazards that are highly publicized by instrument manufactures. Paramount among these are the potential to cause irreparable damage to sight and to inflict serious burns to exposed skin. These hazards are particularly serious if the laser wavelength is outside the visible range since an operator may not realize exposure is taking place until it is too late. The health safety issues outlined above are applicable when using a near-IR laser source for Raman spectroscopy.

Recent Raman analysis instruments show a preference for using near-IR sources because interference from sample florescence is less severe than for visible lasers. Other advantages include the availability of stable, reliable, and affordable diode laser sources and wavelength compatibility with CCD technology for detection of the scattered Raman radiation. As discussed below, another less publicized hazard exists when using near-IR lasers for analysis based on interaction of the laser radiation with the sample.

This report concerns itself with safety issues other than the health hazards associated with skin and eye exposure mentioned above, and focuses on safety issues that arise due to irradiation of the sample with the intense Raman excitation source. Raman is often touted as being a nondestructive analytical technique. This is not always the case. The 785-nm 300-mW diode laser, the source used in the InPhotonics RS2000 instrument, operates at a sufficient power to burn samples. This is especially true if the sample tends to absorb rather than scatter the excitation radiation. This little advertised fact is of particular interest to investigators wishing to analyze irreplaceable samples (forensic evidence, fine art, or in vivo medical diagnostics) or hazardous energetic materials (flammable solvents, explosives, and initiators).

A number of electronic literature searches were conducted on Chemical Abstracts through STN International. A search of the key words "Raman" and safety" crossed with "explosive or explosives" yielded only 4 references since 1975 that specifically identify Raman analysis as a causative factor in initiating explosive decomposition of samples [1-4]. The small 
number of incidences reported in the literature emphasizes that the potential to detonate energetic materials during Raman analysis is not a widely known phenomenon.

The emphasis of our studies is to generate guidelines for the safe Raman analysis of hazardous materials, including military explosives, shock-sensitive explosives, mixtures containing explosives, flammable solvents in bulk solution or on absorptive backgrounds, and gunpowders. The study is largely phenomenological and experimentally progresses from a thermographic investigation of different surface colors, to examining the hazardous materials mentioned above with the goal of extracting the critical parameters important for safe Raman analysis.

This report is divided into 4 main experimental sections. The first studies focus on thermography of colored surfaces. This study investigates the tendency of the 785-nm Raman laser to heat various colors on an extensive color chart. The second section investigates safety issues related to Raman laser use when analyzing flammable organic solvents. The third section of this report examines the ability of the Raman laser to initiate deflagration of explosives (or mixtures that contain explosives) and propellants. The fourth and final experimental section of this report describes deflagration studies with a variety of gunpowders including smokeless, black, and Pyrodex powders. After the experimental sections are presented, the report concludes with a summary that gives recommendations based on the experimental work presented.

\section{$\underline{\text { Experimental Studies }}$}

\section{Heat Transfer to Colored Surfaces}

\section{Experimental Design and Set-up}

A color chart that was constructed for Internet web design was obtained from VisiBone (Sunrise, Florida). This poster chart has 216 colors contained in 16 columns and 16 rows. Each color has three different unambiguous color designations. These identifiers are 1) a unique anglocentric name, 2) a hexadecimal HTML color-code, and 3) a decimal RGB color-code. In addition to the full sized poster charts, 8" x 11.5" laminated color chart replicates are available and would be convenient for field use. One of these laminated charts is included with this report. An electronic representation of the VisiBone color chart is presented in Figure 1. The poster 


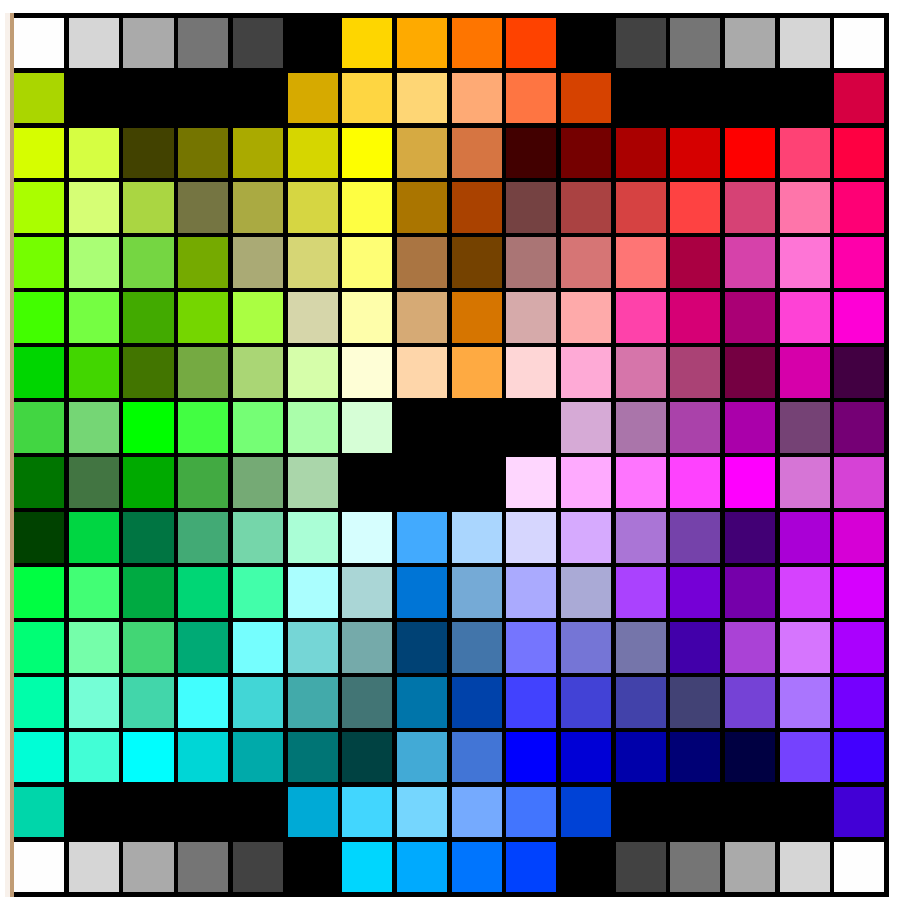

Figure 1. VisiBone color chart representation.

and 8" x 11.5" laminated card have the three different color identifiers located on each color patch. Figure 2 only displays the color and the correct position of the color in the VisiBone chart without reference to the specific color identifiers.

To determine the extent that the 785-nm Raman laser source heats each individual color patch, we considered several different experimental approaches. In general it was considered best to illuminate the color patch with defocused laser light and measure the surface temperature of the illuminated patch. A defocused beam was necessary since it is known from past studies that the focussed beam was capable of thermally damaging dark-colored surfaces. The most desirable experimental arrangement to accomplish the temperature measurements would be to use an IR camera. The Raman laser probe could be placed a sufficient distance away from the color patch to project an approximate 4-mm diameter illuminated spot on the chart surface. A crude shutter would then be rigged to allow the laser to illuminate the color patch starting at a prescribed time. IR photographs would then be taken as the illuminated spot heats up to thermal equilibrium. 
Several preliminary tests were conducted before attempting the IR imaging experiment. First, a black portion of the VisiBone poster was irradiated at different distances from the probe surface to determine the distance range that caused surface damage (pitting). Black was chosen for this experiment since this color was expected to heat the most when irradiated with the laser. It was found that the poster paper was pitted only when the Raman probe was within about $2 \mathrm{~mm}$ of its $1.0-\mathrm{cm}$ focal length.

To project a sufficiently large spot to allow for thermal imaging, it was judged that the probe should be place at about $2.0-\mathrm{cm}$ from the target; or at twice the focal length from the probe face lens. This would project an approximate 4-mm spot onto the color patch surface. In addition to providing a sufficiently large area to collect thermal imaging data, the defocused laser spot minimized surface damage of the color patches due to excessive heating as described above. Next, columns from the VisiBone chart were cut into strips for the experiment. Gloves were worn while preparing and handling the strips to avoid contaminating the surface with fingerprints. Next, the strips were mounted on an optical stage using base clamps as shown in Figure 2. Thermal insulation was provided between the aluminum stage and the color strip by inserting a heavy piece of cardboard (poster board) between the aluminum stage and the color strip.

Figure 3 shows the complete experimental arrangement. The optical stage containing the color strip was mounted vertically with a standard optical mount. The Raman probe was positioned $2.0-\mathrm{cm}$ away from the strip at about $30^{\circ}$ off axis (measured from the perpendicular). This angle was required to allow an unobstructed photographic path to the strip so that temperature could be recorded. In between the strip and the Raman probe, a simple shutter mechanism was fashioned from an aluminum plate. The shutter strip was normally in a position that blocks the Raman probe laser beam. To start an experiment, the shutter was simply swung to the side manually. The camera was positioned directly in front of, and perpendicular to, the color strip. The camera was a Cincinnati Electronics IRRIS-256ST Infrared Radiometric Imaging System (Mason, $\mathrm{OH}$ ) that detects energy in the 3 to 5 micron wavelength range. On the other side of the camera, a laser presentation pointer $(670 \mathrm{~nm})$ was positioned to highlight the center of the spot illuminated by the Raman laser. Alignment of the laser pointer was made through the IR camera with the Raman laser impacting the color strip. The alignment laser 
allowed for rapid and accurate color patch positioning while the near-IR Raman probe beam was shuttered.

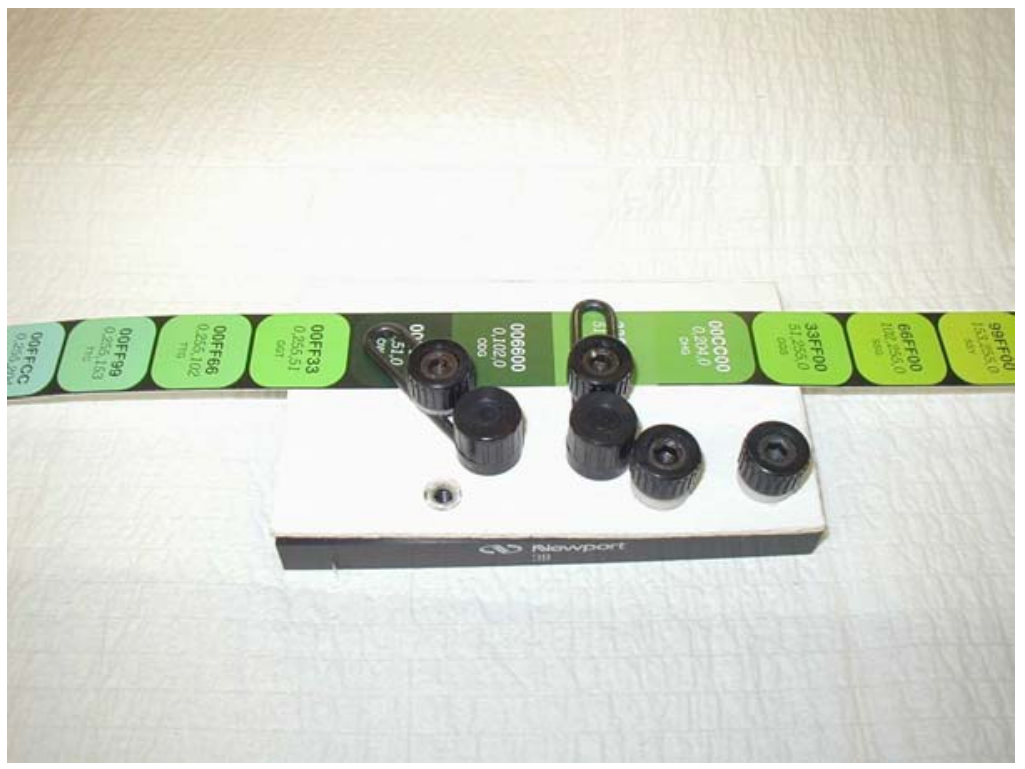

Figure 2. VisiBone color chart strip mounted in the insulated optical mount.

Figure 3. Experimental set up for the IR thermography experiment (see text for complete description). 
Before the experiment was begun, the emissivity of the chart paper was tested relative to a black body emitter. The experimental set up to measure emissivity is shown in Figure 4. A color strip (the far left VisiBone color strip column) was used for the emissivity determination. After temperature equilibration, an IR photograph of a black body emitter was taken alongside the color strip. The black body was a thermally insulated 4" diameter copper pipe elbow that had its interior surface painted with flat black paint. An emissivity of 1.00 in the 3-5 micron wavelength range was indicated by equivalent temperatures measured at three different observation points along the length of the color strip and the black body. The emissivity value of 1.00 resulting from this experiment was used for temperature determinations.

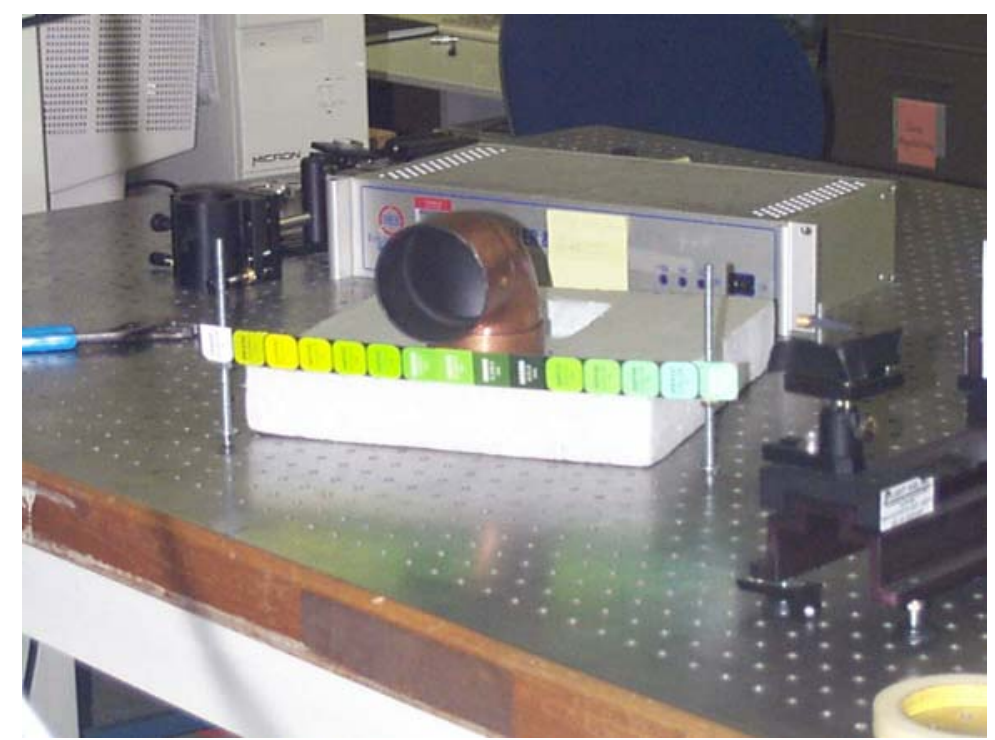

Figure 4. Apparatus used for the determination of emissivity of the VisiBone color strip.

Preliminary range finding experiments helped define the final conditions chosen for the experiment. For these experiments a black color patch was again chosen as representative for a color that would undergo extreme heating. Although the 3 to 5 micron spectral range of the IR camera was well outside 0.785 -micron wavelength of the laser, it was unknown whether the intense Rayleigh scattering would interfere with temperature measurements. Initial temperature determinations showed that this concern was unfounded, as interference was not observed. Next, the black color patch was irradiated for a period of 15 seconds while photographs were examined 
PNNL-13942

every second. The temperature quickly rose initially but appeared to level off after about 8 seconds of illumination. Based on this result, the final experimental protocol recorded temperatures once every second for 15 seconds. The laser shutter was opened 2 seconds into each run so that the last IR image for each color represents 13 seconds of laser irradiation. This time should be sufficient for all irradiated color spots to come to thermal equilibrium. Different temperature ranges of the camera were used during the experiment to cover a broad range of temperatures. If the camera became saturated during an experiment, the procedure was repeated on a higher CCD camera detector range.

\section{$\underline{\text { Time Course Experiment }}$}

The thermal imaging experiment proceeded for all 216 colors as described above. For each color, data was collected to plot temporal temperature profiles to show how rapidly each color heated upon irradiation with the Raman laser. Data for several representative color patches are shown in Figure 5. In general, the color patches reach a temperature plateau well before 13 seconds. Additionally, it was observed that dark colors showed a clear tendency to achieve higher temperatures during irradiation.

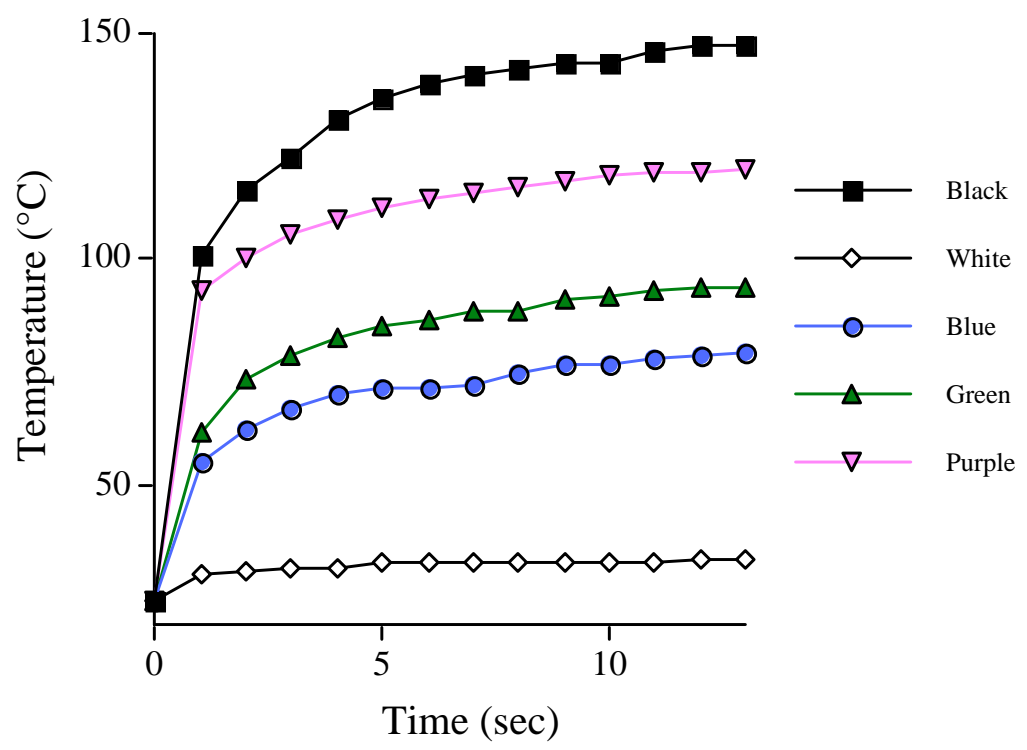

Figure 5. Representative temporal profiles for various colors. 


\section{Thermal Imaging Results}

A summary of the thermal imaging results is presented in Table 1. This table presents the final temperatures in degrees Centigrade achieved for each VisiBone color patch after 13 seconds of laser irradiation. The temperature positions correspond to color positions presented in Figure 1. Locations marked by an "X" indicate blank positions in the color chart.

Table 1. Final temperatures in degrees Centigrade reached after 13 seconds of exposure to the defocused Raman laser. Temperature positions match the color patch positions presented in Figure 1.

\begin{tabular}{|r|r|r|r|r|r|r|r|r|r|r|r|r|r|r|r|}
\hline 33.2 & 72.7 & 112.6 & 141.3 & 142.8 & 146.9 & 29.5 & 34.6 & 30.4 & 29.9 & 146.9 & 142.8 & 141.3 & 112.6 & 72.7 & 33.2 \\
\hline 75.6 & $\mathrm{X}$ & $\mathrm{X}$ & $\mathrm{X}$ & $\mathrm{X}$ & 62.3 & 37.9 & 61.1 & 54.6 & 29.1 & 64.0 & $\mathrm{X}$ & $\mathrm{X}$ & $\mathrm{X}$ & $\mathrm{X}$ & 80.2 \\
\hline 42.6 & 46.4 & 134.4 & 128.1 & 99.0 & 71.2 & 35.5 & 64.2 & 56.1 & 140.3 & 130.5 & 125.9 & 82.2 & 30.5 & 31.8 & 30.0 \\
\hline 55.6 & 43.6 & 78.4 & 127.7 & 94.2 & 63.2 & 30.9 & 95.0 & 96.1 & 134.3 & 115.1 & 76.3 & 28.7 & 77.2 & 28.7 & 29.7 \\
\hline 66.7 & 56.8 & 113.0 & 106.1 & 95.2 & 65.0 & 49.6 & 93.1 & 123.3 & 117.1 & 73.8 & 28.2 & 115.9 & 53.8 & 29.0 & 29.5 \\
\hline 78.8 & 65.0 & 114.0 & 77.0 & 51.1 & 60.2 & 54.9 & 56.5 & 50.8 & 71.2 & 28.0 & 29.0 & 77.5 & 104.2 & 29.6 & 30.1 \\
\hline 93.8 & 91.9 & 129.5 & 108.0 & 77.2 & 27.2 & 69.3 & 38.4 & 39.2 & 27.6 & 28.1 & 72.8 & 120.3 & 129.8 & 53.8 & 142.6 \\
\hline 93.6 & 89.4 & 81.2 & 80.3 & 70.4 & 51.5 & 77.4 & $\mathrm{X}$ & $\mathrm{X}$ & $\mathrm{X}$ & 71.2 & 115.2 & 117.4 & 118.7 & 130.3 & 132.5 \\
\hline 129.7 & 133.7 & 114.0 & 114.2 & 108.6 & 78.2 & $\mathrm{X}$ & $\mathrm{X}$ & $\mathrm{X}$ & 27.5 & 28.6 & 29.2 & 29.8 & 30.1 & 76.2 & 75.3 \\
\hline 137.2 & 76.3 & 126.0 & 90.6 & 78.8 & 41.6 & 61.9 & 38.1 & 53.8 & 38.3 & 37.9 & 84.7 & 122.7 & 133.5 & 80.9 & 74.0 \\
\hline 65.9 & 70.5 & 104.8 & 71.5 & 62.3 & 38.8 & 82.8 & 62.4 & 71.1 & 52.9 & 86.8 & 45.0 & 70.5 & 120.6 & 42.2 & 41.2 \\
\hline 68.2 & 53.6 & 76.9 & 94.4 & 36.3 & 65.9 & 93.4 & 123.7 & 104.3 & 65.5 & 99.1 & 126.2 & 123.5 & 79.3 & 42.1 & 55.8 \\
\hline 57.0 & 47.2 & 66.7 & 33.0 & 69.5 & 97.2 & 94.0 & 94.8 & 93.7 & 72.8 & 108.6 & 136.0 & 133.9 & 86.5 & 56.8 & 67.4 \\
\hline 42.0 & 54.4 & 62.8 & 67.0 & 109.5 & 128.2 & 123.4 & 69.0 & 73.5 & 78.5 & 110.4 & 125.1 & 140.9 & 149.8 & 70.7 & 76.2 \\
\hline 68.6 & $\mathrm{X}$ & $\mathrm{X}$ & $\mathrm{X}$ & $\mathrm{X}$ & 71.4 & 68.1 & 53.2 & 35.8 & 71.0 & 88.7 & $\mathrm{X}$ & $\mathrm{X}$ & $\mathrm{X}$ & $\mathrm{X}$ & 80.6 \\
\hline 33.2 & 72.7 & 112.6 & 141.3 & 142.8 & 146.9 & 77.0 & 39.5 & 56.1 & 79.3 & 146.9 & 142.8 & 141.3 & 112.6 & 72.7 & 33.2 \\
\hline
\end{tabular}

To allow an easily interpreted visual representation of the heating associated with each color, the patches susceptible to heating in Table 1 were converted into colors that represent relative hazard. This simplified hazard interpretation is presented in Figure 6. Values used in the calculations for this representation were 1) the minimum final temperature observed $\left(28.0^{\circ} \mathrm{C}\right)$ during the experiment, 2) the maximum final temperature $\left(146.8^{\circ} \mathrm{C}\right)$, and 3$)$ the difference between the minimum and maximum temperatures achieved $\left(118.8^{\circ} \mathrm{C}\right)$. Relative hazard values were calculated by taking the difference between each color patch final temperature and the minimum temperature $\left(28^{\circ} \mathrm{C}\right)$, multiplying this number by 10 , and then dividing this value by the maximum observed temperature range $\left(118.8^{\circ} \mathrm{C}\right)$. This calculation results in a value between 1 and 10. Figure 6 indicates the thermal hazards as increasing from yellow (relative hazard of 7.5 to 8.4 ), through orange (relative hazard of 8.5 to 9.4 ), to red (relative hazard of 9.5 to 10.0 ) 
which represents the maximal thermal hazard. Although this assignment of hazard-related colors to specific numerical values is somewhat arbitrary, the presentation allows quick identification of colors that will be most problematic when irradiated with the Raman 785-nm near-IR laser. Comparison of the relative hazards (Figure 6) to the VisiBone color patches (Figure 1) again demonstrate that darker colors are associated with the most propensity for sample heating.

Using the results presented above, a field agent could easily match the color of a sample against the 8" x 11.5" laminated VisiBone chart and then look up the associated thermal hazard associated with analyzing this sample using the 785-nm Raman laser.

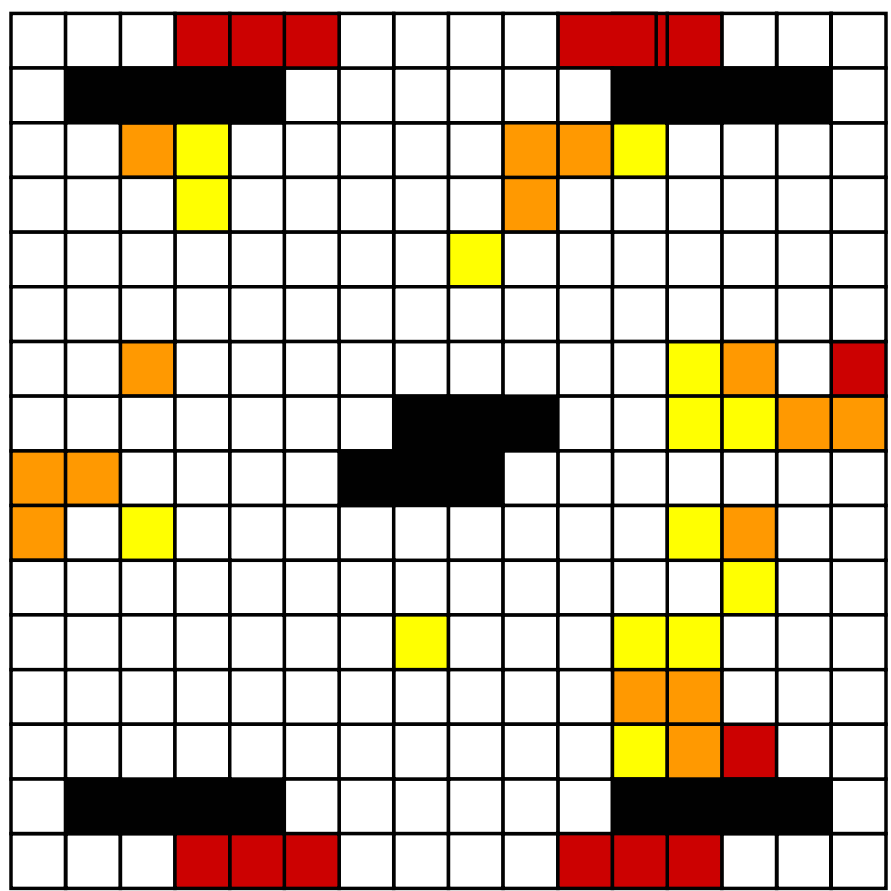

Figure 6. Relative thermal hazard associated with analysis of various colors. Color positions are matched to those in Figure 2. Hazard increases from yellow to red as described in detail in the text. 
PNNL-13942

\section{Solvent Flammability}

\section{$\underline{\text { Preparation for Flammability Experiments }}$}

Several 3.0" x 1.5" x 0.5" aluminum blocks were machined and 2 depressions milled in the top surface with a ball bit (See Figure 7). These blocks were anodized in black as well as dark shades of green, purple, and blue by Quality Coatings, LLC (Post Falls, ID). The machined wells were filled with various solvents and illuminated with the Raman laser to test for solvent ignition. In these experiments, the high thermal conductivity of the aluminum is expected to play an important role in quickly dispersing the surface heat and making solvent ignition less likely than if a material of lower thermal conductivity was used.

To present a worse case scenario for solvent ignition, a dark color in combination with a material of low thermal conductivity was needed. Pyrex glass or white glazed porcelain plates with well depressions, originally designed to observe color forming chemical reactions, were used for these experiments after being coated with a near-IR-absorptive coating. A photograph of the porcelain plates along with the various aluminum blocks is shown in Figure 7. The Pyrex and porcelain substrates were sprayed with Aerodag G, a black graphite aerosol spray product, to create a near-IR absorptive surface layer that was resistant to solvents. Unlike aluminum, the thermal conductivity of Pyrex and porcelain are low; therefore surface heating will remain localized. This scenario will emphasize solvent ignition potential; however, it should be noted that other materials exist that have even lower thermal conductivity, such as wood or plastic, that were not tested in these experiments.

\section{$\underline{\text { Parameters Effecting Solvent Ignition Potential }}$}

There are two practical scenarios where flammable solvent ignition represents a safety concern during Raman analysis. First is the potential for the Raman laser to cause heating in a bulk solvent or in mixtures containing a flammable solvent. The second is related to the analysis of a flammable solvent spill on a surface that may be heated by the Raman laser. These two situations are addressed separately below by experiments that irradiate solvents on various colored backgrounds as well as studies of bulk solvents that contain IR-opaque dyes. 


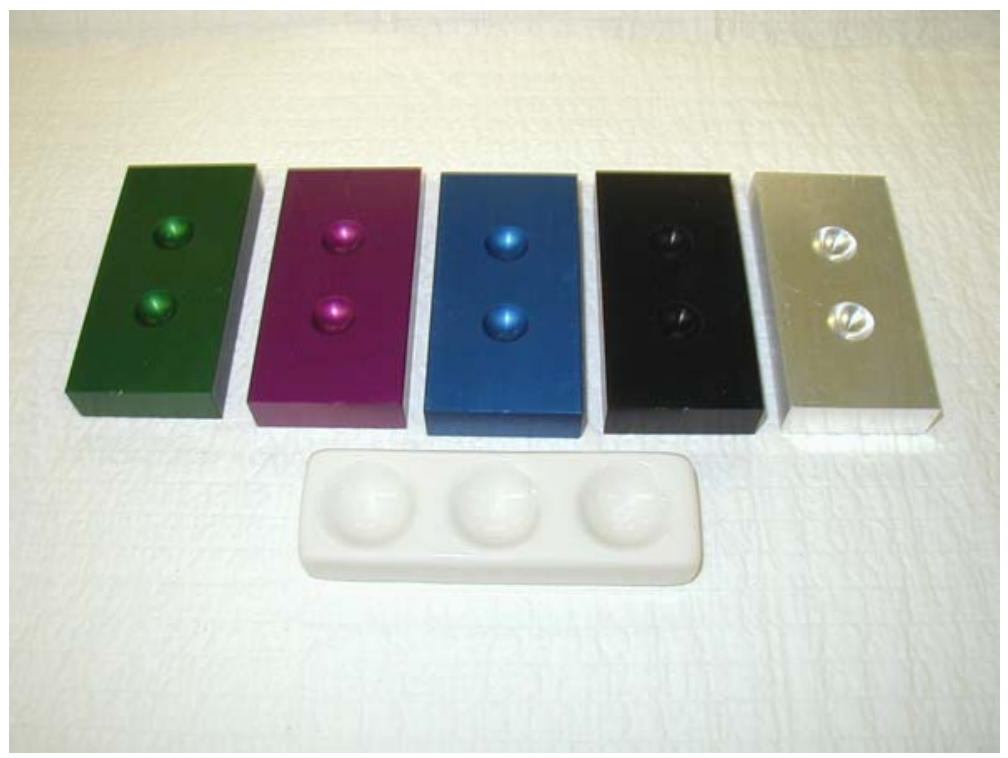

Figure 7. Photograph of blocks with machined wells for the solvent flammability study. Aluminum blocks have either been left untreated or have been anodized in blue, purple green, and black. A white ceramic block is also shown that will be coated in black Aerodag $\mathrm{G}$ before testing occurs.

Several parameters are expected to be important for solvent spills present on colored backgrounds including the near-IR absorptivity of the substrate surface, thermal conductivity of the substrate, and magnitude of cooling due to solvent evaporation. If the background material absorbs near-IR radiation, heat will be generated. The overall significance of the generated heat is largely determined by the thermal conductivity of colored substrate. If the material is able to dissipate the heat effectively, localized temperature increases will not be high and solvent flammability hazards will remain low. On the other hand, for materials that have low thermal conductivity, the heat generated cannot be effectively dissipated and localized temperatures may dramatically rise upon laser irradiation. In these cases, the safety hazard may be high depending on the magnitude of the temperature increase. Another factor that must be considered for volatile solvents is the cooling associated with liquid evaporation. For highly volatile organic solvents the evaporative cooling effects may dominate such that heating effects from laser absorption are negligible.

Experiments that examine colored substrates will focus on three materials; aluminum, glazed-porcelain, and Pyrex glass. Aluminum blocks that contained machined depressions or 
wells were either left untreated or anodized in a variety of dark colors that would be expected to absorb near-IR radiation. Thermal effects generated on aluminum, however, were unlikely to be significant due to the large thermal conductivity $(216.5 \mathrm{~W} / \mathrm{m}-\mathrm{K})$ of this metal. Pyrex and porcelain on the other hand have similar and much lower thermal conductivity values of 1.1 $\mathrm{W} / \mathrm{m}-\mathrm{K}$ and 10-14 W/m-K, respectively. The thermal conductivity range for of porcelain was an estimate provided by the color plate manufacturer (CoorsTek, Golden, CO). Our studies examined these materials after they were coated with a thin film of graphite paint (Aerodag $\mathrm{G}$ ). The graphite strongly absorbs near-IR radiation and, due to the low thermal conductivity of the porcelain or Pyrex, will give rise to maximal temperatures in the laser illumination region. The graphite-coated glass and/or porcelain therefore represent a worst-case scenario. If solvents don't ignite on these materials, ignition on dark colored substrates is not expected to represent a significant safety hazard.

\section{$\underline{\text { Solvent Flammability on Graphite-Coated Surfaces }}$}

The flammable solvents studied on graphite-coated porcelain and Pyrex substrates were diethyl ether, acetone, hexane, isooctane, toluene, gasoline, and diesel fuel. These solvents were chosen to encompass a wide range of volatility and to represent a variety of chemical functional groups. For this experiment 2-5 drops of solvent was placed in the sample well and the laser focussed on the bottom of the well. A microscope slide was placed immediately after the probe face, in between the probe and sample well, to protect the probe optics in case of solvent ignition. Solvent was illuminated at full laser strength until all traces of solvent had evaporated, or for at least 200 seconds for the less volatile solvents. Each observation was repeated in triplicate. The results are summarized in Table 2 and are further discussed below.

Several of the solvents tested had a tendency to dissolve the Aerodag-coated surface. Application of these solvents to the graphite-coated wells resulted in a roughened coating, or in some cases, a small amount of graphite-solvent slurry in the bottom of the well that formed before the last traces of solvent evaporated. Solvents that had the most pronounced effect on the Aerodag coating were acetone and ethanol.

For the more volatile solvents, thermal effects of laser illumination could be observed on the graphite-coated well surface. As the solvent evaporated, the center of the well dried first as a 
result of the laser heating the graphite coating in the bottom. This resulted in a concentric solvent ring forming prior to complete solvent evaporation. Solvents that displayed this thermal effect are designated with a numerical "2" in Table 2. Concentric solvent rings were not observed for toluene and diesel fuel due the low volatility of these solvents. Thermal effects were, however, observed for gasoline. This was due principally to the low surface tension of gasoline on the graphite surface. Instead of remaining in the bottom of the well, gasoline applied to the graphite surface dispersed as a thin layer. This phenomenon made observing localized thermal effects easier.

Table 2. Flammability study results shown in order of increasing solvent boiling point.

\begin{tabular}{|l|l|l|l|l|l|l|l|}
\hline $\begin{array}{l}\text { Solvent/ } \\
\text { Surface }\end{array}$ & $\begin{array}{l}\text { Aluminum } \\
(\mathrm{Al})\end{array}$ & $\begin{array}{l}\text { Anodized } \\
\text { Green Al }\end{array}$ & $\begin{array}{l}\text { Anodized } \\
\text { Purple Al }\end{array}$ & $\begin{array}{l}\text { Anodized } \\
\text { Blue Al }\end{array}$ & $\begin{array}{l}\text { Anodized } \\
\text { Black Al }\end{array}$ & $\begin{array}{l}\text { Graphite } \\
\text { Coated } \\
\text { Pyrex }\end{array}$ & $\begin{array}{l}\text { Graphite } \\
\text { Coated } \\
\text { Porcelain }\end{array}$ \\
\hline $\begin{array}{l}\text { Diethyl } \\
\text { Ether }\end{array}$ & 1 & 1 & 1 & 1 & 1 & 2 & 2 \\
\hline Acetone & 1 & 1 & 1 & 1 & 1 & 2 & 2 \\
\hline Hexane & 1 & 1 & 1 & 1 & 1 & 2 & 2 \\
\hline Ethanol & 1 & 1 & 1 & 1 & 1 & 2 & 2 \\
\hline Isooctane & 1 & 1 & 1 & 1 & 1 & 2 & 2 \\
\hline Toluene & 3 & 3 & 3 & 3 & 3 & 3 & 3 \\
\hline Gasoline & 3 & 3 & 3 & 3 & 3 & 2 & 2 \\
\hline $\begin{array}{l}\text { Diesel } \\
\text { Fuel }\end{array}$ & 3 & 3 & 3 & 3 & 3 & 3 & 3 \\
\hline
\end{tabular}

$\$$ Color plates from CoorTek are a 60-65\% alumina, 30-35\% clay-based silica composite with a silica glaze.

$1=$ Illuminated until evaporated - no effect observed.

$2=$ Illuminated until evaporation - laser thermal effects observed but no solvent ignition.

3 = Illuminated for more than 200 seconds - no effect observed.

During initial observations with hexane, small bubbles could be seen rising from the center of the illuminated solvent-filled cell. Apparently the boiling of point of hexane was exceeded at the solvent-graphite interface and the solvent gently boils in the localized region of the focussed laser. This effect was also observed for isooctane. Bubble formation was not observed for solvents more volatile than hexane, apparently because the evaporative cooling prevented localized temperatures from reaching the boiling point. Bubble formation was not 
observed of solvents less volatile than isooctane since temperatures at the graphite-solvent interface were not sufficient to promote boiling.

The same experiment was repeated on graphite-painted Pyrex for comparison to coated porcelain. This experiment generally gave similar results as the graphite-coated porcelain. One interesting phenomenon was observed on coated Pyrex during the acetone and ethanol experiments. As the solvent evaporated it was possible to move the laser to the edge of the concentric solvent ring and illuminate a small amount of graphite-solvent slurry with the laser to the point where glowing red graphite embers could be observed. Despite the obvious heat being generated, the remaining ethanol or acetone solvent did not ignite. Several reasons could explain why this phenomenon was observed on the coated glass and not the coated porcelain surface. Explanations may include a lower Aerodag-glass adhesion, a possibly thicker glass-graphite coating, and a lower thermal conductivity for glass; these factors favor formation of a more abundant graphite-solvent slurry on glass with less effective heat dissipation.

\section{$\underline{\text { Uncoated Porcelain and Aluminum, as well as Anodized Aluminum Surfaces }}$}

Similar solvent flammability experiments were performed with the plain white porcelain, uncoated aluminum, and aluminum anodized in black, green, purple, and blue colors. As before, 3 to 5 drops of each solvent was placed in a well and illuminated with the laser either until complete evaporation, or for at least 200 seconds for the less volatile solvents. Experiments were carried out in triplicate. Solvent experiments performed on these substrates did not result in ignition or any observable thermal effects.

\section{Bulk Solvent Heating}

For solvent heating experiments we sought a near-IR dye that strongly absorbed in the near-IR and had an absorption wavelength maximum that closely matched the 785-nm laser output. This represents a worst case scenario for bulk solvent heating since conditions are optimal for absorption of laser energy and thermal transfer to the liquid. It is possible that solvent heating could pose a safety hazard if the thermal transfer is pronounced.

To address this issue we purchased several dyes that absorb in the IR region of the spectrum (America Dye Source Inc., Quebec, Canada). One of these dyes, ADS775PP, has a 
maximum absorption band in the near-IR at a region that closely matches the output of the 785nm Raman laser. The spectrum of the dye is presented in Figure 8. The red line in Figure 1 represents the Raman laser output wavelength. ADS775PP has an absorption maximum in methanol at $780 \mathrm{~nm}$ and an absorption coefficient of $2.60 \times 10^{5} \mathrm{~L} / \mathrm{mole}-\mathrm{cm}$. This dye was mixed in solvent at several concentrations to asses the heating potential of an absorptive organic liquid. For studies that probed solid explosive mixtures (described in a later section), we used an additional dye that partially absorbs the laser wavelength. This dye, ADS818HI, has an absorption wavelength maximum of $818 \mathrm{~nm}$ in methanol and a molar absorption coefficient of $2.43 \times 10^{5} \mathrm{~L} / \mathrm{mole}-\mathrm{cm}$.

Initially we wanted to perform these experiments in either hexane or diethyl ether because of the notorious flammability associated with these solvents. The ADS775PP dye, however, was not very soluble in either of these solvents. On the other hand, the manufacture's literature states that this dye has a high solubility in methanol $(65 \mathrm{mg} / \mathrm{ml})$ and we adopted this organic solvent for our studies based principally on solubility issues. The control experiment examined $3.0 \mathrm{ml}$ of methanol in a 4.0-ml vial that was capped with a septum sealed screw-cap top. A K-type thermocouple was inserted through the septum and positioned in the vial just below the solvent meniscus and adjacent to the glass wall. The Raman laser was focused into the

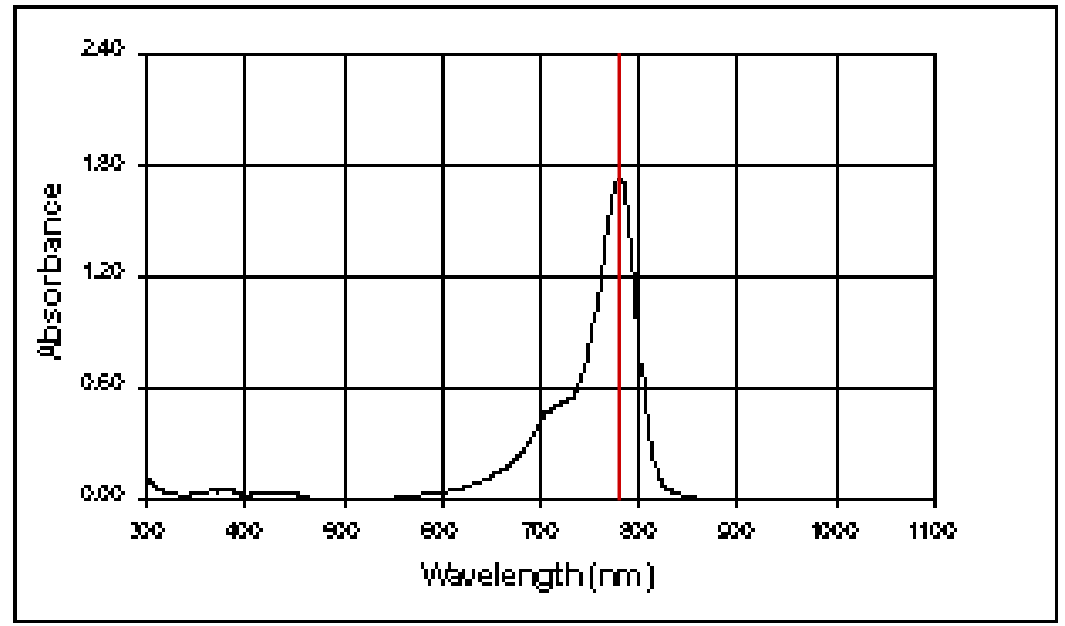

Figure 8. Spectrum of ADS775PP dissolved in methanol showing a close match between the dye absorbance maximum at $780 \mathrm{~nm}$ and the laser wavelength at $785 \mathrm{~nm}$. 
center of the methanol solution at a position well below the thermocouple level. The laser was turned on and temperature measurements taken every 15 seconds for an elapsed time of 10 minutes.

For comparison to the control, 3.0-ml solutions that contained either $3.0 \mathrm{mg} / \mathrm{ml}$ or 9.0 $\mathrm{mg} / \mathrm{ml}$ of ADS775PP dye were examined. The experimental conditions were identical to the control. The results of this study are presented in Figure 9. The $3.0 \mathrm{mg} / \mathrm{ml}$ solution showed a classic heating curve characterized by rapid initial heating followed by thermal equilibrium being reached by the end of the 10 -min exposure. This dye solution showed a preferential $2.5^{\circ} \mathrm{C}$ heating over the methanol control. The solution that contained $9.0 \mathrm{mg}$ dye $/ \mathrm{ml}$ methanol heated faster and did not reached thermal equilibrium by the end of the 10-min exposure. This solution heated $5.6^{\circ} \mathrm{C}$ more than the methanol control after 10 -min of illumination. It should be emphasized that thermal effects were relatively small and, for reasons stated below, would not be expected to cause safety concerns. First, thermal transfer to the liquid was optimized by using a dye that had a high molar absorptivity and an absorption maximum that coincided with the laser wavelength. Second, the relative heating effects observed were not high (no more than a $5.6^{\circ} \mathrm{C}$ difference from the control). Finally, it is unlikely an acquisition time as long as 10 min would be actually be used; 3 min represents the maximal time a sample is likely to be illuminated during a Raman spectral acquisition.

In a related experiment, a small amount of the 3.0-mg ADS778PP/ml methanol solution was placed in a porcelain well and illuminated as the dye concentration increased due to solvent evaporation. This experiment was also repeated with methanol:diethyl ether $(1: 3, \mathrm{v} / \mathrm{v})$ that was saturated with the ADS775PP dye. Although direct focussing of the laser on neat ADS775PP dye will cause the material to degrade and smoke, there was no indication of solvent ignition during these evaporative concentration experiments. 
PNNL-13942

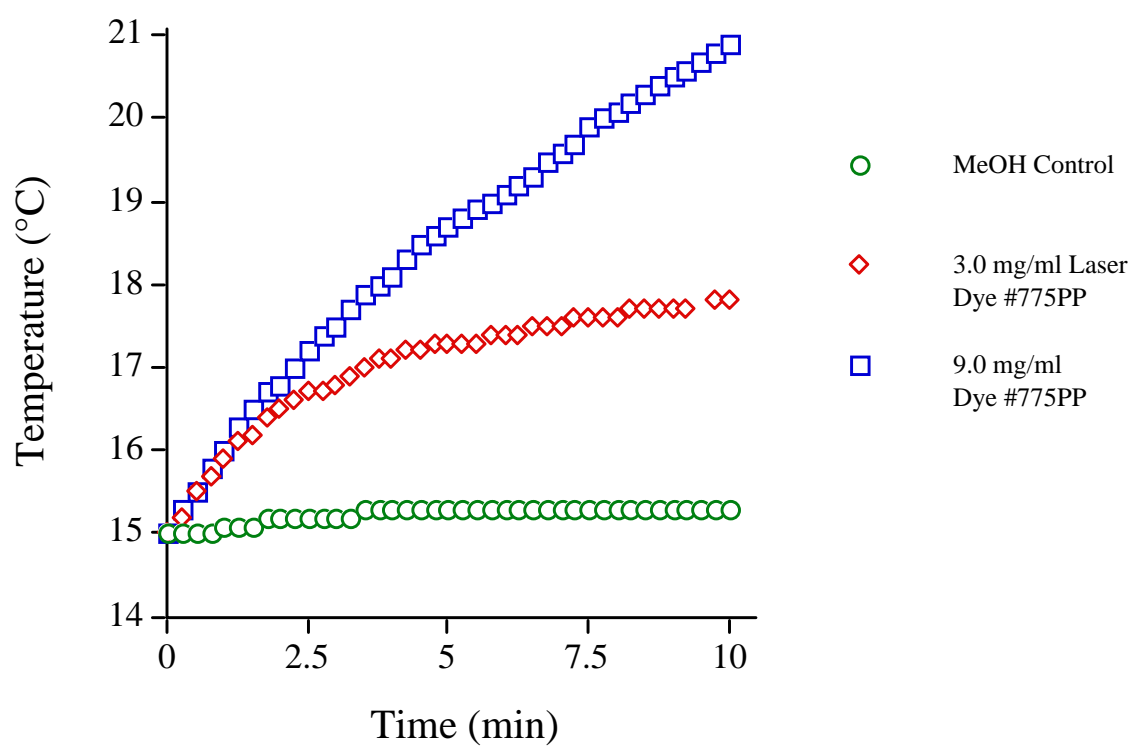

Figure 9. Solution heating caused by 785-nm Raman laser irradiation of a methanol solution containing two concentrations of a near-IR dye.

\section{Deflagration of Explosives and Propellants}

\section{$\underline{\text { Propellants }}$}

Two propellants that are used in intercontinental ballistic missiles were used for this study. The first was a black-colored single-base propellant from the first stage of a Minuteman missile, whereas the second was a double-based green-colored propellant from a sea-launched Polaris missile. Both propellants were obtained from Thiokol Propulsion (Brigham City, UT). When the IR laser was focussed on the single-base material, localized ignition was generated as evidenced by small sparks in the region of the focussed laser beam; however, the reaction remained localized and did not result in sustained ignition. For the double-base propellant, the focussed laser elicited wisps of smoke from the surface although, again, sustained ignition did not occur. 


\section{Military Explosives}

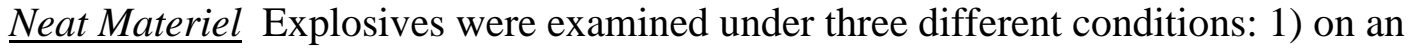
aluminum surface, 2) on a graphite-coated porcelain surface, and 3) on a background of black construction paper. Military-grade high explosives were obtained directly from military sources. Explosives examined were 2,4,6-trinitrotoluene (TNT), $N$-2,4,6-tetranitro- $N$-methylanaline (tetryl), hexahydro-1,3,5-trinitro-1,3,5-triazine (RDX), octahydro-1,3,5,7-tetranitro-1,3,5,7tetrazocine (HMX), butanetrioltrinitrate $(\mathrm{BTTN})$, and pentaerythritol tetranitrate (PETN). The results of these experiments are described below and summarized in Table 3.

Table 3. Propensity of military explosives to deflagrate when illuminated with a 785-nm Raman laser source on various surfaces.

\begin{tabular}{|l|l|l|l|l|l|l|}
\hline $\begin{array}{l}\text { Conditions/ } \\
\text { Explosive }\end{array}$ & TNT & Tetryl & RDX & HMX & BTTN & PETN \\
\hline Aluminum & Nothing & Nothing & Nothing & Nothing & Nothing & Nothing \\
\hline $\begin{array}{l}\text { Graphite-Coated } \\
\text { Porcelain }\end{array}$ & Nothing & Nothing & Nothing & Nothing & Nothing & Nothing \\
\hline $\begin{array}{l}\text { Black Construction } \\
\text { Paper }\end{array}$ & $\begin{array}{l}\text { Defla- } \\
\text { gration }\end{array}$ & $\begin{array}{l}\text { Defla- } \\
\text { gration }\end{array}$ & $\begin{array}{l}\text { Defla- } \\
\text { gration }\end{array}$ & Nothing & Nothing & Nothing \\
\hline
\end{tabular}

For experiments involving aluminum or graphite-coated porcelain surfaces, small amounts of explosives (5-10 mg) were placed in the plate wells and illuminated with the full intensity of the focussed laser for periods of approximately $5 \mathrm{~min}$. Deflagration did not occur for any of the explosives during this experiment. This is particularly significant for the off-colored materials since these would be most likely to absorb the Raman laser wavelength. Colored materials included tetryl (light cream-colored pellets), TNT (beige plates), and BTTN (dark orange liquid).

The next experiment examined small amounts of explosives on a black construction paper background. For this experiment, the construction paper was attached to the surface of a $\mathrm{X}-\mathrm{Y}-\mathrm{Z}$ micropostioning stage and the Raman laser was held in a stationary mount above the stage. When focussed on the paper, the laser would burn the paper forming a small pit. The pit 
indicated the precise area and distance where optimal laser focussing occurred. Next, small amounts of explosives placed into and around the paper crater, the laser turned on, and the distance between the probe and the stage slowly decreased until the laser was focussed directly on the explosive. The effect of this procedure was to maximize background heating from the construction paper. The positioning stage was stopped at the laser focal length and allowed to sit for 3-5 min while the illuminated explosive was observed.

Results indicated that RDX grains in the immediate vicinity of the focussed laser would deflagrate on black construction paper. Similarly, one could induce deflagration of PETN on construction paper as indicated by visible sputtering when the laser was focussed on a sparsely dispersed power. TNT would melt upon illumination and could be coaxed to deflagrate after extended illumination. It should be emphasized that although it is possible to induce deflagration of these explosives, it is moderately difficult to do so. Deflagration occurs only on particles directly impacted by the laser on the black paper background and is not self sustained burn. We were not able to induce deflagration of HMX or BTTN. To avoid adsorption of the BTTN liquid on construction paper, the paper was attached to the positioning stage with a single layer of transparent scotch tape. The BTTN was deposited on the tape rather than directly on the paper. When BTTN was illuminated, a red ember could be ignited on the paper beneath a drop of the explosive; however, the BTTN was not effected. This is an indication of how recalcitrant these explosive can be when illuminated with the laser.

Mixtures Containing Explosives Two separate experiments were conducted to probe the sensitivity of near-IR absorptive mixtures containing explosives. The first experiment examined binary solid mixtures that contained $50 \%$ by weight ADS775PP dye mixed with RDX, HMX, tetryl, TNT, or PETN. The second experiment examined mixtures of RDX that contained $27 \%$ by weight of various matrix materials that would be expected to absorb the 785-nm laser wavelength. For both experiments the explosive-containing mixture or controls were placed in a porcelain color plate well and the laser focused on the surface of the sample. A glass slide was placed after the probe, as in previous experiments, to protect the probe optics. In both experiments a careful side-by-side comparison was performed between the explosive-containing mixtures and the relevant control materials. The results of the experiment are summarized in Table 4. 
In interpreting the results presented below, it becomes necessary to rank the magnitude of the laser-induced reaction. For this purpose, the formation of a sustained visible red ember was considered more of an indication of reaction than smoke alone. Further, copious smoke was considered a more vigorous reaction compared to the formation of a small amount of smoke; however, plentiful smoke was not considered as strong a reaction as formation of a visible sustained ember.

As summarized in Table 4, all the dye/explosive mixtures exhibited some degree of smoking and discoloration similar to that observed for the control sample (neat dye). The protective glass slide was replaced often during this experimental progression since a residue would readily deposit on the protective glass slide resulting in decreased laser intensity being delivered to the sample. Explosive mixtures all showed enhanced reaction over the control ADS775PP dye. These reactions were subdued with the single exception of PETN. For example, TNT flake could be observed to melt and deflagrate in a very gentle low-intensity manner. As emphasized in Table 4, the mixture with PETN was the most vigorous deflagration and this mixture almost, although not quite, achieved a sustained burn.

Table 4. Tendency of various military explosives mixed with ADS775PP to deflagrate when exposed to a 785-nm Raman laser source.

\begin{tabular}{|l|l|}
\hline Mixture & Observation \\
\hline ADS775PP Control & Turns black and smokes, no ember visible \\
\hline ADS775PP:RDX (50:50, w/w) & Can urge a short-lived ember \\
\hline ADS775PP:HMX $(50: 50, \mathrm{w} / \mathrm{w})$ & Can urge a short-lived ember \\
\hline ADS775PP:tetryl $(50: 50, \mathrm{w} / \mathrm{w})$ & $\begin{array}{l}\text { Can observe deflagration of individual } \\
\text { tetryl pellets }\end{array}$ \\
\hline ADS775PP:TNT $(50: 50, \mathrm{w} / \mathrm{w})$ & $\begin{array}{l}\text { Observe melting and deflagration of } \\
\text { individual TNT flakes }\end{array}$ \\
\hline ADS775PP:PETN $(50: 50, \mathrm{w} / \mathrm{w})$ & $\begin{array}{l}\text { Most vigorous of all - almost a sustained } \\
\text { burn but not quite; copious smoke }\end{array}$ \\
\hline
\end{tabular}

Next, six mixtures that contained RDX were prepared with different matrices that would be expected to absorb the 785-nm laser wavelength. In addition to the ADS775PP dye, we also used a dye for this experiment that partially absorbs the 785-nm laser wavelength. The 
ADS818HI dye, as previously mentioned, has an absorption maximum at $818 \mathrm{~nm}$ in methanol and a molar absorption coefficient of $2.43 \times 10^{5} \mathrm{~L} / \mathrm{mole}-\mathrm{cm}$. Another matrix material used in this experiment was the carbon-based chromatographic molecular sieve adsorbent material Carboxen (Supelco, Bellefonte, PA). Carboxen consists of relatively large $(0.49 \pm 0.04 \mathrm{~mm}$ diameter, $n=6$ ) black spherical particles. Yet another matrix material examined was relatively large hollow iron oxide spheres $(0.85 \pm 0.06 \mathrm{~mm}, \mathrm{n}=6)$. A soil was included in this experiment since soil mixtures with RDX are likely to be encountered in the field. We used a forest loam soil (Cinebar) that has a high organic carbon content.

The matrix mixture experiments used a higher proportion of RDX (73\% by weight) in an attempt to make deflagrations more obvious. Results for this experiment are summarized in Table 5. In all cases, RDX-containing mixtures show a more vigorous reaction than the control matrix. With the single exception of iron oxide, however, the reactions were very mild. Iron oxide mixed with RDX showed a sustained deflagration that consumed practically all the material placed in the well. The specific iron oxide used in this experiment consisted of relatively large spherical particles. Interestingly, Carboxen, a material that also consisted of relatively large spherical particles, also exhibited an obvious deflagration when mixed with RDX. Large spheres tend to act as a unit when irradiated to form a large glowing ember. RDX powder coats the larger particles and more readily deflagrates as the coated sphere is heated. The matrix materials that consisted of small-particle powders (such as graphite) interspersed with the RDX were less likely to give a rigorous reaction because less RDX overall is exposed to the glowing ember upon irradiation as compared to the larger particles (like the iron oxide or Carboxen above).

Due to the vigorous reaction observed above with iron oxide, more extensive experiments were conducted with mixtures of finely divided powders of iron oxide and aluminum, either separately or together with RDX. Iron oxide and aluminum, when combined in equal amounts (on a weight basis), form the pyrophoric mixture known as thermite. The results of irradiating the aluminum, iron oxide, and thermite powders, both with and without RDX, are shown in Table 6. Results lend credence to the previous observations that particle size plays an important role in determining deflagration intensity. Iron oxide powder combined with RDX will exhibit deflagration of RDX, but not in a sustained manner as was seen previously for the larger 
Table 5. Propensity of mixtures of RDX with various matrices to deflagrate when exposed to a 785-nm Raman laser source.

\begin{tabular}{|l|l|}
\hline Mixture or Control & Observation \\
\hline 1. Iron oxide:RDX $(15: 40, \mathrm{w} / \mathrm{w})$ & Sustained vigorous deflagration/burn \\
\hline Control - Iron Oxide & $\begin{array}{l}\text { Particles glow red with an occasional spark } \\
\text { observed }\end{array}$ \\
\hline 2. Carboxen:RDX $(15: 40, \mathrm{w} / \mathrm{w})$ & $\begin{array}{l}\text { RDX-coated ember deflagrates but short } \\
\text { lived since reaction blows other particles } \\
\text { out of laser path }\end{array}$ \\
\hline Control - Carboxen & Red glowing embers \\
\hline 3. Graphite powder:RDX $(15: 40, \mathrm{w} / \mathrm{w})$ & Smoke due to RDX deflagration \\
\hline Control - Graphite Powder & Small ember, no smoke \\
\hline 4. Cinebar Soil:RDX $(15: 40, \mathrm{w} / \mathrm{w})$ & $\begin{array}{l}\text { Continuous localized "burning" ember - far } \\
\text { more vigorous than control }\end{array}$ \\
\hline Control - Cinebar Soil & Hot spot embers as move laser over surface \\
\hline 5. ADS775PP:RDX (15:40, w/w) & $\begin{array}{l}\text { Copious smoke, more than seen for control; } \\
\text { no ember observed }\end{array}$ \\
\hline Control - ADS775PP & Continuous smoke; no ember seen \\
\hline 6. ADS818HI:RDX (15:40,w/w) & $\begin{array}{l}\text { Ember that "burns"; more vigorous than } \\
\text { control }\end{array}$ \\
\hline Control - AD818HI & Continuous smoke; no ember seen \\
\hline
\end{tabular}

Table 6. Tendency of RDX mixed with aluminum, iron oxide and thermite powders to deflagrate when illuminated with a 785-nm Raman laser source.

\begin{tabular}{|l|l|}
\hline Mixture or Control & Observation \\
\hline Aluminum: RDX $(15: 40, \mathrm{w} / \mathrm{w})$ & $\begin{array}{l}\text { Faint ember; difficult to induce glowing; } \\
\text { less than control; no smoke }\end{array}$ \\
\hline Control - Aluminum & Medium intensity ember; no smoke \\
\hline Iron Oxide:RDX $(15: 40, \mathrm{w} / \mathrm{w})$ & $\begin{array}{l}\text { Glows brightly; not steady; harder to } \\
\text { induce glow than control but sputters and } \\
\text { smokes indicating RDX deflagration }\end{array}$ \\
\hline Control - Iron oxide & Bright high intensity steady ember \\
\hline Thermite:RDX & $\begin{array}{l}\text { Sparks and very bright flashes; similar to } \\
\text { control }\end{array}$ \\
\hline Control - Thermite & $\begin{array}{l}\text { White bright flashes as move laser across } \\
\text { surface }\end{array}$ \\
\hline
\end{tabular}


particles of iron oxide. Aluminum with RDX did not show reaction over the control, in fact, it was harder to induce a glowing ember in the RDX mixture due to the "dilution" of aluminum powder with RDX. An interesting phenomenon was observed when thermite was illuminated. Bright flashes were produced as the focussed laser was moved across the thermite surface indicating partial ignition of this material. Ignition, however, was not self sustained. The observations were similar regardless of whether the RDX was present in the thermite mixture or not.

\section{$\underline{\text { Shock-Sensitive Explosives }}$}

Nitrogen Triiodide The first step in synthesis of this contact explosive was to recrystallize iodine to obtain small crystals rather than the large plate crystals that are usually encountered. Small crystals enhance product yield by increasing the reactive surface area of the iodine starting material. Iodine was recrystallized by dissolving in a small quantity of refluxing methanol and then combining this solution with a larger quantity of water at $0^{\circ} \mathrm{C}$. The iodine crystals were collected by vacuum filtration in a medium sintered glass funnel. The vacuum was then removed and the recrystallized iodine submerged in concentrated ammonium hydroxide and allowed to react in the funnel for $5 \mathrm{~min}$. Vacuum was then applied and the nitrogen triiodide product collected. The moist product was divided into wells on porcelain plates and allowed to dry.

The reaction can be followed by the subtle color change from the dark purple color of iodine to the black color of the nitrogen triiodide product. Nitrogen triiodide is ideal for these studies because of its extreme shock sensitivity and color that is ideal to absorb the near-IR laser wavelength. The dried material was carefully positioned below the Raman laser such that the laser, once it was powered up, was focussed directly on the explosive surface. A microscope slide again was positioned between the probe and the explosive to protect the optical components during detonation. The laser power was turned on and slowly increased. Nitrogen triiodide detonated well before full laser power was reached. This experiment was repeated a second time with similar results. This second trial was videotaped to capture the laser-induced detonation.

$\underline{\text { Trinitroglycerin }}$ Trinitroglycerin represents an extremely unstable shock sensitive explosive. Trinitroglycerin is a colorless liquid that should be relatively transparent toward near- 
IR radiation. For these experiments we purchased nitroglycerin standards from Radian International (Austin,TX). These standards are available in 1.0-ml ampoules at a concentration of 1.0-mg trinitroglycerin/ml acetonitrile. For our experiments we pooled 7 ampoules and evaporated the acetonitrile solvent to approximately 500- $\mu$ l under a stream of nitrogen. The 500$\mu \mathrm{l}$ solution was transferred to a porcelain well and the remainder of the acetonitrile allowed to evaporate. The remaining nitroglycerin was illuminated with the full power of the Raman laser for a period of 5 min without any observed effects. At this point, the trinitroglycerin was taken up with about $500 \mu \mathrm{l}$ of acetone, transferred to an aluminum well, the solvent allowed to evaporate, and the explosive was illuminated; however, this time a spectrum was taken in order to demonstrate that the laser was focussed on the explosive. The spectrum of trinitroglycerin obtained during this experiment is presented in Figure 10. Other than the obvious lack of laserinduced detonation, this spectrum is noteworthy in that it represents a spectrum taken from only $7 \mathrm{mg}$ of trinitroglycerin. The final experiment involved dissolving the trinitroglycerin in acetone and transferring to a graphite-coated porcelain well. Due to the near-IR absorptive properties of graphite, illumination on this substrate is much more likely to trigger detonation than on uncoated porcelain. Consistent with studies on the other background materials, illumination of trinitroglycerin on graphite-coated porcelain with the near-IR Raman laser did not result in detonation.

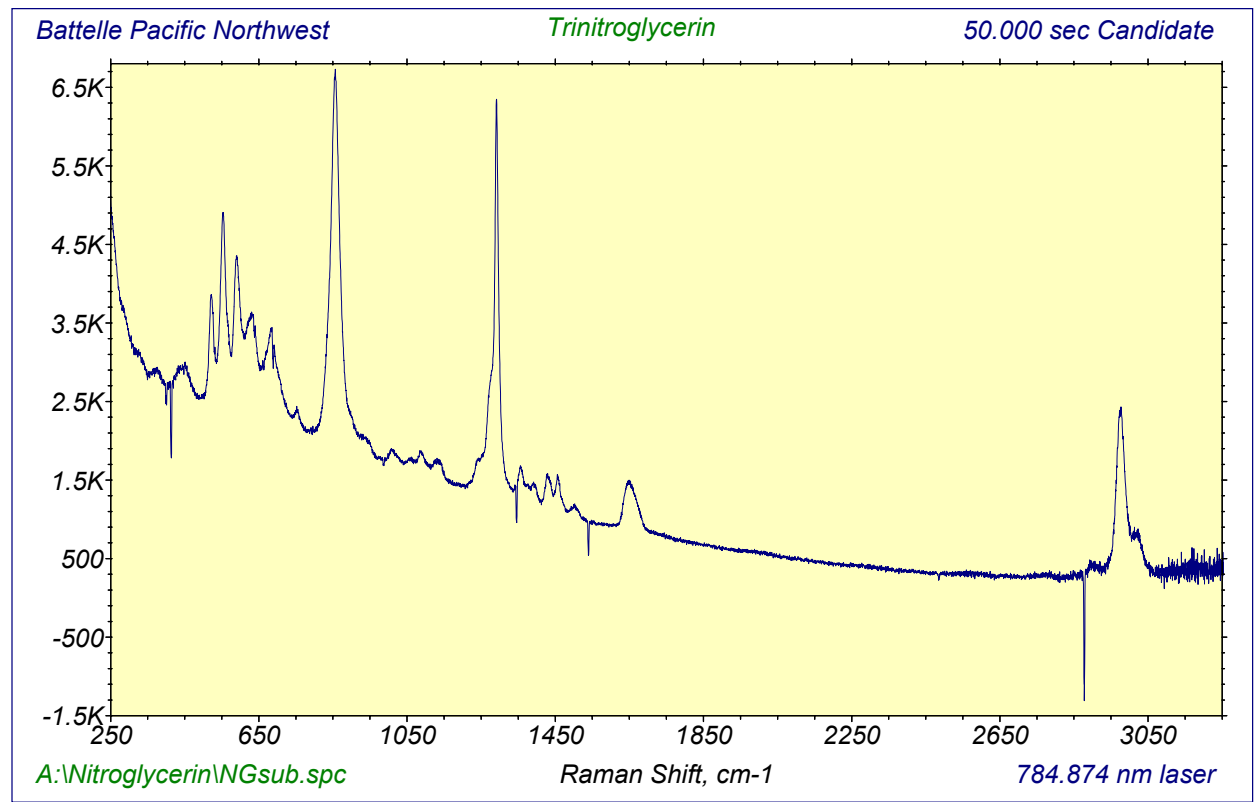

Figure 10. Raman spectrum of trinitroglycerin taken from 7.0-mg of material contained in an aluminum well. 


\section{Deflagration of Gun Powders (Smokeless, Black, and Pyrodex Powders)}

Our previous experiments examined a variety of smokeless powders and, for us, was the first indication that the Raman laser could ignite certain samples. The purpose of the present study was to verify our initial findings and expand these studies to black and Pyrodex powders. For the smokeless powder class, a variety of powders having different burn rates were included in the experiment. In order of decreasing burn rate, we examined red dot, green dot, and DuPont \#4198 powders. The "dot" series are flake powders, whereas the DuPont material is a cylindrical canister powder that has a relatively fast burn rate (although nowhere near as fast as red or green dot). The burn rate of black and Pyrodex powders is controlled by particle size; the smaller particles burn at a higher rate. Particle sizes for various powders are given in Table 7. Within either the black or Pyrodex powder classes, the materials have a fairly homogenous chemical composition. Therefore, our experiments can look for differences between particle sizes within a powder class as well as for differences in ignition sensitivity between smokeless, black, and Pyrodex powders.

Table 7. Different powder designations and particle sizes.

\begin{tabular}{|l|l|l|}
\hline Powder Class & Designation & Particle Size Diameters \\
\hline Smokeless & Green Dot & Disks $=\sim 1.4 \mathrm{~mm}$ \\
\hline Black & Fg & 1.68 to $1.19 \mathrm{~mm}$ \\
\hline Black & FFg & 1.19 to $0.59 \mathrm{~mm}$ \\
\hline Black & FFFg & 0.84 to $0.297 \mathrm{~mm}$ \\
\hline Black & FFFFg & 0.42 to $0.149 \mathrm{~mm}$ \\
\hline Pyrodex & FFg Equivalent & 1.19 to $0.59 \mathrm{~mm}$ \\
\hline Pyrodex & FFFg Equivalent & 0.84 to $0.297 \mathrm{~mm}$ \\
\hline
\end{tabular}

For this experiment the Raman probe was positioned in a stationary holder above an XY-Z micro positioner. A microscope slide placed in front of the Raman probe to protect the probe optics from fouling during powder ignition. A uniform thin layer of powder was deposited on an aluminum stage directly below the Raman probe. An experiment was initiated by turning the laser to full power and then slowly decreasing the distance between the probe and the powder 
to the point where powder ignition occurred. The distance between the stage and the Raman probe was then measured with a micrometer. Each powder was examined a total of four different times.

The results are shown graphically in Figure 11. The ordinate values start at $10 \mathrm{~mm}$ since this distance is the focal point of the Raman probe. In other words, this is the distance where the laser power density will be highest. The further the distance from the probe where ignition occurs, the more sensitive the material is toward 785-nm laser illumination. Error bars in Figure 11 represent the standard deviation of the distance measurements. The results clearly show that smokeless powders were much more sensitive toward ignition than the Pyrodex or black powders. Higher sensitivity of the smokeless relative to the black and Pyrodex powders can also be presented in terms of power density required to initiate deflagration. From the average ignition distances for the smokeless class compared to the combined black and Pyrodex groups, one can calculate that the smokeless powder requires only 0.64 the power density to initiate deflagration. Although complex factors may contribute, this enhanced sensitivity is most likely attributable to the trinitroglycerin base of the smokeless powders. Trinitroglycerin may be brought to the surface as the powder is heated by the Raman laser. Other factors that might contribute include possible differences in IR-absorptivity between the powders. Within the smokeless class, there did not seem to be any pronounced differences between powders with different burn rates. Likewise, there were no obvious differences in the ignition sensitivity between black or Pyrodex powders or any clear differences between particle sizes within the black or Pyrodex powder classes.

Although smokeless powder is more sensitive to ignition, it should be mentioned that once ignition occurs, the smokeless flake powder combustion is not that vigorous. Usually, one or two of the "dot" flakes will sputter and ignite only a few adjacent flakes; however, the entire powder layer set up for the experiment did not combust. Ignition of the DuPont cylindrical canister powder was even less robust than the flake powders. In contrast, ignition of Pyrodex or black powder was violent and combustion was all or nothing. 
PNNL-13942

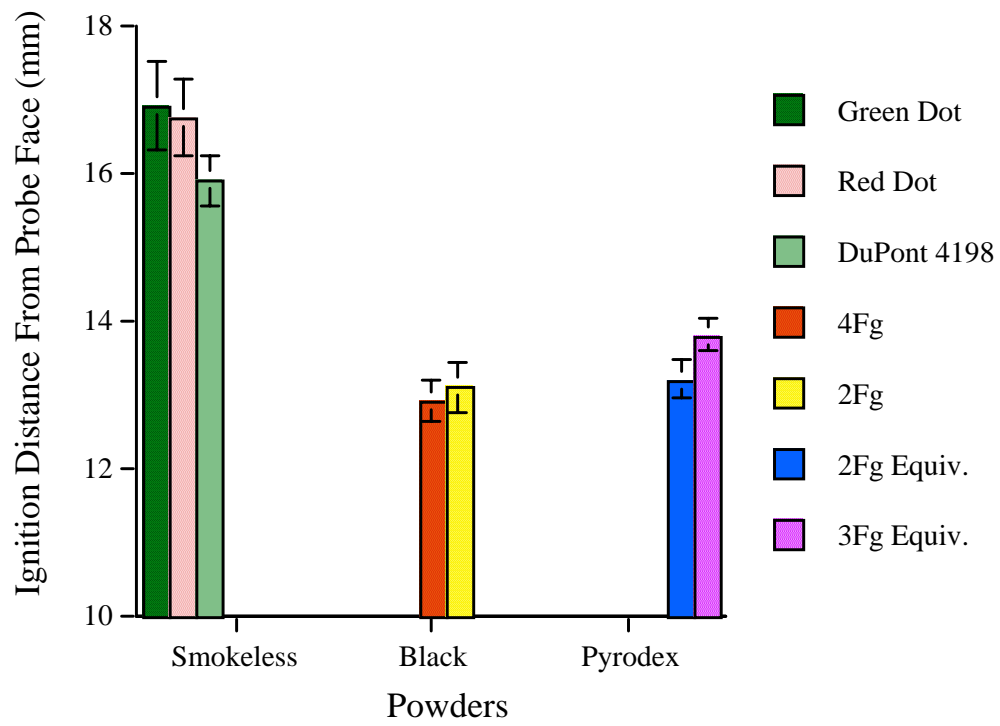

Figure 4. Ability of 785-nm Raman laser to ignite smokeless, black, and Pyrodex powders (see text for details).

\section{Conclusions and Recommendations}

Thermography studies presented in the first portion of this report give an indication of the amount of heat that is generated on different visible color surfaces by the near-IR laser. The visible spectrum is usually considered to range from 400 to $800 \mathrm{~nm}$. Safety projections based on the thermography studies are not perfect since colors we observe in the visible range are not ideally correlated with absorption characteristics in the near-IR. However, given the proximity and slight overlap of the laser excitation wavelength to far red portion of the visible spectrum, these studies are valid and give the best approximation for predicting the thermal response an unknown sample when exposed to the Raman laser source.

The thermography studies demonstrate that dark colors absorb the 785-nm laser wavelength resulting in sample heating. Dark colored materials are most likely to cause problems during analysis not only due to the high thermal safety risks but also because poor quality spectra are likely to result due to a preference for laser absorption rather than Raman 
scattering. Relative hazards associated with analysis of an unknown can be rapidly assessed by matching the unknown sample color to the laminated VisiBone color chart and then referencing Table 1 and Figure 6 in this report to determine the heating hazard. This will give a reasonable idea of how safe an unknown sample is to interrogate.

Studies presented in this report assessed the safety of analyzing flammable organic solvents on dark backgrounds. Since solvent ignition was not observed and thermal effects were minimal, we conclude that the safety risks are low. This is not to say, however, that the risk is non-existent. Pyrex glass was the lowest thermal conductivity material we studied, yet there exist other materials with lower thermal conductivity such as wood and plastic. These materials may allow more rapid heating of the solvent and higher safety risks. Additionally, our studies only examined nonporous surfaces. It is possible that porous materials that are saturated with flammable solvents would behave differently than the surfaces we studied. Because all secenarios could not be experimentally addressed, it is prudent to isolate small amounts of solvent containing surface material for analysis in case ignition does occur. If the situation dictates a subsample cannot be taken, and sufficient potentially flammable material is present to endanger the operator, the experiment should be triggered remotely, if at all.

From experiments performed with bulk solvents that contain a near-IR absorptive dye we conclude that although heat is transferred to the sample, the temperature increase is limited and the procedure is safe. This is provided that adequate liquid is present to serve as a heat sink (as a general guideline volume should be $1 \mathrm{ml}$ or greater) and the laser illumination period is limited to less than $10 \mathrm{~min}$.

Deflagration experiments showed that self-sustained burns could not be achieved for propellants although these material were observed to smoke (in the case of the double-based material) and spark (in the case of the single base material). We conclude it is safe to analyze these materials if it is possible to limit the amount used for an experiment to less than approximately $50 \mathrm{mg}$. It is acknowledged that this recommendation is based on observations from only a limited number of propellants.

For neat military explosives, our experiments indicate that is safe to analyze these materials except when they are situated on a black background. We observed deflagration of a 
few explosives on black construction paper but combustion was listless and not self sustained. A shortcoming of this recommendation is that in real-life situations, it is impossible to know $a$ priori if the material is neat or a mixture. Experiments of explosives mixed with a near-IR dye, and of RDX mixed with matrix materials, gave an occasional sample that yielded a vigorous burn when illuminated with the Raman laser. In our experiments, the dye/PETN combination almost achieved a self-sustained burn. The iron oxide pellet mixture with RDX was the only mixture in our studies that gave a sustained deflagration. Follow-on studies seemed to indicate that the large particle size of the iron oxide was an important parameter that determined deflagration intensity. Regardless, the occasional explosive mixture that yields a self-sustained deflagration is extremely important from a safety standpoint. Recommendations that emerge from this study again make reference to the VisiBone color chart. The color of iron oxide is associated with a high-risk area of the VisiBone color chart (an orange square in Figure 6). If unknown materials have colors that are associated a pronounced tendency to heat, then only small amounts of material should be interrogated.

Studies also examined a few shock sensitive explosives. Nitrogen triiodide (black color) consistently detonated when impacted with the laser even when the laser intensity was muted, whereas trinitroglycerin (clear) did not exhibit any ill effects even when illuminated at full laser power for long periods of time. These studies emphasize the primary importance sample color plays in the safety associated with analysis.

Final studies examined gunpowders. This group exhibited the most predictable deflagrations of any group of materials we studied. Our experiments gave the interesting result that smokeless powders are more sensitive toward ignition than black or Pyrodex powders. Smokeless powders, although more sensitive toward laser-induced ignition, gave generally listless deflagrations. This burning behavior contrasted sharply with the rapid and complete combustion of black and Pyrodex powders. All the gunpowder materials were a characteristic black or dark gray color. These colors correspond to the most hazardous areas delineated by the thermography experiments; these areas are shaded red in Figure 6. The recommendation is that black or dark gray materials should not be analyzed under any conditions. 
Several untested ideas have been suggested on how to avoid gunpowder (or other sample) deflagration. One suggestion is to use a laser that operates at a different wavelength. For example, one of the most intense lines from an argon-ion laser (either 514.5- or 488-nm) could be used for the Raman experiment. The argon-ion laser would certainly have different sample absorption characteristics than the 785-nm near-IR laser. For gunpowder though, it is not clear that this would avoid the problem of heat transfer to the sample since black samples absorb all visible wavelengths. It is reported that a $514.5-\mathrm{nm}$ (green) unfocussed $200-\mathrm{mW}$ beam from an argon-ion laser is capable of catching a black card on fire. Using this laser source for Raman analysis of black pyrophoric samples would not be expected to be successful. Additionally, substitution of a visible laser would increase interference due to sample fluorescence. Other untested suggestions include using either a pulsed laser or chopped laser beam to minimize sample heating. 
PNNL-13942

\section{References}

1. S. Kuehling, H. Keul, and H. Hoecker, Poly(2,4-hexadiyn-1,6-ylene carbonate). Synthesis and topochemical cross-linking reaction, Macromolecules, 23:4192-4195 (1990).

2. C.J. Nielsen, P. Klaeboe, H. Priebe, S.H. Schei, The vibrational spectra, molecular structure and conformation of organic azides. Part III. 2,3-Diazido-1,3-butadiene, J. Mol. Struct., 147(3-4):217-229 (1986).

3. N.C. Craig and M.A. Kliewer, Raman spectrum of difluorodiazirine, Spectrochim. Acta, Part $A$, 35A(7):895-897 (1979).

4. J.E. Griffiths, W.A. Sunder, and W.E. Falconer, Raman spectra of the dioxygenyl and nitrosyl fluorometalate salts O2+MF6-, O2+M2F11, and NO+MF6-, M = As, Sb, Bi, Nb, Ta, Ru, Rh, Pd, Pt, Au, Spectrochim. Acta, Part A, 31A(9-10):1207-1216 (1975). 